Proceedings

\title{
Sensor-Based Smart Oven System to Enhance Cooking Safety ${ }^{\dagger}$
}

\author{
Rami Yared * and Bessam Abdulrazak * \\ Department of Computer Science, University of Sherbrooke, 2500 boul. de l'Université, Sherbrooke J1K 2R1, \\ QC, Canada \\ * Correspondance: rami.yared@usherbrooke.ca (R.Y.), bessam.abdulrazak@usherbrooke.ca (B.A.) \\ + Presented at the 3rd International Electronic Conference on Sensors and Applications, \\ 15-30 November 2016; Available online: https://sciforum.net/conference/ecsa-3.
}

Published: 14 November 2016

\begin{abstract}
The recent advances in sensor technology empower adaptable smart systems targeting safety. Smart sensing in ambient intelligence systems enables to enhance safety during cooking which is very important for aging people. Therefore, we worked on a project of building a smart oven system. We studied the principal risks around oven and analyzed the characteristics and the basic functional principles of the existing sensors to select the most appropriate. In this paper, we present the analysis of the sensors used and the test results of each sensor in a real-world cooking environment.
\end{abstract}

Keywords: sensors; smart oven; infrared; ultrasonic; hygrometer; electrochemical; metal oxide semiconductor; cooking risks; safety; fire; burn; intoxication; gas; smoke; ambient technology

\section{Introduction}

The recent advances in sensor technology enable to build adaptable context-aware smart systems for diverse uses [1]. Ambient intelligence systems based on smart sensing enhance safety while performing Activities of Daily Living (ADL) particularly for aging people. Aging people face several risks while performing indoor and outdoor ADL [2-4]. Cooking is an essential indoor ADL. This issue is particularly critical for those with cognitive decline that usually forget the cooking unattended. Studies revealed that unattended cooking is the main leading factor responsible for fire in the kitchen $[5,6]$ and the kitchen is the second place where the majority of indoor fire accidents occur, and in particular the oven presents the main source of fire accidents in residences $[5,7]$. Advanced sensors enable contextual data acquisition around oven. We present in this paper our analytical study related to sensors selection for our smart oven system.

The paper is structured as follows: Section 2 discusses the related work on sensors used to prevent risks in the kitchen. Section 3 presents our research methodology for the sensors selection. Section 4, details the analytical study of the sensors selection, as well as the test results of each sensor in a real-world cooking environment. Section 5 concludes the paper and discusses future directions.

\section{Related Work}

Most of the existing sensor-based systems to prevent risks in kitchen focus on fire risk. An existing basic solution to detect fire risk at home is installing fire alarms. However, the existing fire alarms have several drawbacks. They require replacing alarm batteries regularly. In addition, they generate false alarms (e.g., in the presence of a small quantity of smoke generated by regular cooking). Lushaka et al. use smoke alarms in their system to detect a potential fire risk [6]. The system considers only fire risk and depends on smoke alarms. A number of studies mention oven monitoring as a part of larger systems to track activities of daily living. Alwan et al. [8] measure oven usage and 
Wai et al. [9] propose detecting unsafe usage of the oven. They use embedded temperature sensors to measure the burner status, ultrasonic sensors to detect the presence of a pot and electric current sensors to detect the oven utilization and the levels of abnormality in the kitchen. They require modifications to oven to install the sensors. Yuan et al. develop an automated top oven monitoring system based on thermal cameras to detect dangerous situations [10]. The system does not require modifications to oven, so it fits any existing oven and respects user privacy, because it is based on thermal imaging and not a visible-light camera. However, the thermal camera is sensitive to cooking heat and smoke. To build a robust smart oven system, it is required to insightfully select sensors.

\section{Research Methodology}

One important aspect of building a smart oven system to enhance cooking safety is to select appropriate sensors for contextual data acquisition. Therefore, we started by a study of the risks during cooking. We identified the major risks associated with cooking as fire, burn by splash and by contacting hot objects, and intoxication by gas/smoke [11]. Then, we established the relation between the contextual parameters and triggering risks. As a summary, the determined pertinent parameters for each risk are: Fire: we observed the concentrations of Volatile Organic Compound (VOC), and Alcohol gases in the cooking smoke; Burn: for burn risk by splash and by contacting hot objects, we observed relative humidity, utensil temperature, burner temperature, and presence of a utensil on a burner; and Intoxication by gas/smoke: we observed the concentration of Carbon monoxide (CO) gas in the cooking smoke. The analytical study related to kitchen risks drove the selection of sensors to monitor and measure each of the identified parameters. We mainly analyzed the characteristics of the existing sensors in order to select the most appropriate for contextual data acquisition around oven. We also integrated and tested the selected sensors in a real-world cooking environment to determine the capacities/limitations of each sensor. The list of the selected sensor technologies includes: infrared to measure burner and utensil temperatures, ultrasonic to detect the presence of a utensil on oven burner, resistive hygrometer to measure the relative humidity, electrochemical to measure the concentration of $\mathrm{CO}$ gas, metal oxide semiconductor to measure the concentrations of VOC and Alcohol gases in the cooking smoke. Following, we present in details the analytical study related to the sensors selection.

\section{Sensors Selection Analytical Study and Tests}

Sensors are required to satisfy certain functional requirements in order to be integrated as a contextual data acquisition component in an efficient real-world smart oven system. Sensors must be easily installed and integrated (e.g., on a sensor node) to allow practical utilization. For building an efficient smart oven, sensors are required to have short response time to enhance the performance of the whole system. To be deployed in a real-world environment, the price of the sensors has to be taken in consideration. In addition, resistivity to cooking environment (temperature fluctuations, humidity, and the smoke of the cooking process) is an important factor for sensors selection. Following, we present in details our study of these sensors.

\subsection{Sensors Selected for Fire and Intoxication by Gas/Smoke Risk Detection}

The traditional solutions to detect fire are based on smoke and gas detectors $[5,6]$.

\subsubsection{Smoke Detectors for Fire Detection}

The existing common smoke detectors are photoelectric and ionization.

(A) Photoelectric smoke detector: The most common types of photoelectric sensors use a source of light. The emitted light ray is spread in presence of smoke. When the spread light reaches a photoelectric cell, the device detects a smoke and the alarm is triggered.

$(-)$ The photoelectric technology has been excluded because: it is more sensitive to smoke with large particles (particles composed of a big number of molecules "thick smoke") since these particles better spread light rays. However, photoelectric smoke detectors are not sensitive to detect fire 
producing flames (few large particles in the smoke). Therefore, photoelectric smoke detectors are not convenient for detecting a fire risk situation during cooking.

(B) Ionization smoke detector has an ionization chamber and a source of ionizing radiation which is a radioactive source of $\alpha$ particles. The ionization chamber consists of two plates. The battery applies a voltage to the plates and an electric current is produced. When smoke particles enter the ionization chamber, they attach to the ions and neutralize them, so they do not reach the plate. The drop in the electric current between the plates triggers the alarm. The ionization smoke detector is sensitive to smoke with small particles "thin smoke" which corresponds to fire generating flames.

(-) The ionization technology has been excluded because: false alarms are relatively high since the alarm may be triggered in presence of several materials (e.g., cigarette smoke, dust, water droplet). In addition, ionization smoke detectors are prohibited in many countries because of using radioactive materials which is dangerous for health. Consequently, we exclude ionization smoke detectors from the selection criteria.

\subsubsection{Gas Sensors for Fire Detection}

Detection of fire risk is possible by measuring the concentrations of VOC and Alcohol gases in the cooking smoke. The common existing technologies to measure these concentrations include: non-dispersive infrared, metal oxide semiconductor and electrochemical gas sensors.

(C) Non-Dispersive Infrared (NDIR) gas sensor targets the wavelength absorption of the infrared rays as a way to identify particular gases. Therefore, the NDIR sensors can discriminate gases according to their absorbing infrared spectrum. The output of a sensor is an electric voltage proportional to the quantity of gas.

(-) NDIR has been excluded because NDIR sensor output drift (biased) over time and thus it requires recalibration. In addition, this Technology is expensive.

(D) Metal Oxide Semiconductor (MOS) gas sensor: When the MOS material is heated to $\left(400{ }^{\circ} \mathrm{C}\right)$, the oxygen of the air is adsorbed on the metal oxide surface. With its high electron affinity, adsorbed oxygen attracts free electrons inside the metal oxide, forming a potential barrier (high resistance). When the sensor is exposed to a combustible gas the oxidation reaction of such gas with adsorbed oxygen occurs and the sensor resistance decreases. VOC and Alcohol gas concentrations in the air can be detected by measuring the resistance change.

(+) The MOS technology has been selected based on the following reasons: (1) it works without contact which allows convenient integration in a sensor node; (2) and installing the sensor away from the cooking workspace does not disturb user movement; and (3) low price. Additionally, the selected sensors MICS 5521 (e2v technologies, Chelmsford, United Kingdom) and TGS 2620 (Figaro, USA) have a low response time (between $15 \mathrm{~s}$ and $20 \mathrm{~s}$ ).

As a summary of testing MICS 5521 and TGS 2620 while cooking several kinds of food in normal situations: the maximal output voltages are around: (a) $1000 \mathrm{mV} \mathrm{(} \mathrm{60} \mathrm{parts} \mathrm{per} \mathrm{million} \mathrm{ppm)} \mathrm{when}$ cooking fish, onion and peppers in a frying pan; (b) $1500 \mathrm{mV}(\sim 75 \mathrm{ppm})$ when cooking hotdogs in a frying pan; and (c) $2000 \mathrm{mV} \mathrm{(} 100 \mathrm{ppm})$ when heating $50 \mathrm{ml}$ of oil in a frying pan (for $8 \mathrm{~min}$ ) because heated oil releases more VOC and Alcohol in the cooking smoke compared to cooking meat, which releases more VOC and Alcohol than cooking fish and vegetables. Our experimental results revealed that the output voltages of these sensors are above $3000 \mathrm{mV}(\sim 300 \mathrm{ppm})$ in fire risk situations.

\subsubsection{Gas Sensors for Intoxication Detection}

Intoxication risk detection is possible by measuring the concentrations of $\mathrm{CO}$ gas in the cooking smoke.

(E) Electrochemical gas sensor operates by reacting with a specific gas and producing an electrical current proportional to the gas concentration. A typical electrochemical gas sensor consists of two electrodes: anode and cathode separated by a thin layer of electrolyte (an ion conductor). Electrochemical gas sensor operates like a battery with gas being the active material for reaction.

By measuring the current between the two electrodes, this electrochemical cell can be utilized as a 
gas sensor. Electrochemical gas sensors are sensitive to fluctuations of temperature and humidity and also sensitive to parasite gases that may react with the electrodes. Therefore, most of the existing electrochemical gas sensors integrate two filters: one for humidity and the second for other gases which makes electrochemical gas sensors convenient to reduce false alarms when parasite gases exist in the environment.

(+) The electrochemical technology has been selected based on the following reasons: (1) it works without contact which allows convenient integration in a sensor node; (2) and installing the sensor away from the cooking workspace does not disturb user movement; (3) low price. Additionally, the selected sensor TGS 5042 (Figaro, USA) has an acceptable response time (between $15 \mathrm{~s}$ and $60 \mathrm{~s}$ ).

As a summary of testing TGS 5042 while cooking several types of food in a frying pan: the sensor measurements show that the CO concentration is around $40 \mathrm{ppm}$ in normal situations and around $800 \mathrm{ppm}$ in intoxication risk situations.

\subsection{Sensors Selected for Burn Risk Detection}

Detection of burn by contacting hot objects is possible by measuring the temperatures of a utensil and the burner. In order to detect a burn by splash, the system has to start tracking the variations of the relative humidity of the surrounding environment when a utensil is on the oven burner. Therefore, burn by splash detection is possible by measuring the relative humidity and the presence of a utensil on the burner. The common existing technologies to measure these parameters include: infrared (for temperature), resistive hygrometer (for humidity), and ultrasonic (for presence of a utensil on the burner by measuring the distance between a utensil and the sensor).

(F) Passive infrared temperature sensor measures the temperature of an object based on the infrared rays emitted by the object. The sensor has a specific field of view to detect the emitted infrared rays by an object.

(+) The infrared technology has been selected to measure utensil and burner temperatures because it works without contact which allows convenient integration in a sensor node and for its reasonable price.

As a summary of testing the selected MLX 90614 (Melexis, Ypres, Belgium): the measurements of an object temperature mainly vary according to: the infrared emissivity of the object, the position of the sensor (distance between the sensor and the object and the field of view of the sensor), and the size of the object. We found certain imprecision in the measurements of utensil temperature due to the low infrared emissivity of the metals and to the heat produced by the cooking process that disturb the measurements of the infrared sensor. Therefore, we performed the necessary calibrations to overcome the sensor measurement imprecision.

(G) Relative humidity hygrometer sensor is based on a sensible material placed between two electrodes to measure the relative humidity. The impendence of the hygrometer varies according to the relative humidity of the environment and the variations are transformed to electrical voltage variations such that the output voltage is proportional to the relative humidity.

$(+)$ The hygrometer technology has been selected because it could be installed remotely from the monitored zone.

As a summary of testing the selected HIH-5030 sensor (Honeywell, New Jersey, USA) while heating water using different kinds of utensils: the hygrometer starts to react immediately when steam is released. The sensor measurements reflected the variations of the relative humidity while water is boiling.

(H) Ultrasonic distance measurement sensor emits a sound pulse and the reflected sound is then received by the sensor. The detection of the sound generates an output signal which can be analog or digital.

$(+)$ The ultrasonic technology has been selected because it measures the distance of target objects using non-contact technology and it is widely used.

As a summary of testing the selected SRF02: the ultrasonic wave propagation varies with surrounding air temperature. Each time there is a hot air between the sensor and a utensil, the measurement becomes less reliable. This is the case when a utensil is not placed in the center of 
the burner, or the case when a utensil is smaller than the burner. We compensate the imprecision in sensor measurements by defining a confidence zone $[12,13]$ such that, if a utensil is placed in the interior of this zone, it is considered to be on the burner.

\subsection{Discussion}

The technologies of the selected sensors (i.e., electrochemical, metal oxide semiconductor, infrared, ultrasonic, and resistive hygrometer) do not require contact to operate, hence they can be easily integrated on a sensor node and installed around the cooking workspace without interfering with user movement. The analog output signals of the selected sensors are easy to acquire. In addition, the reasonable price and the appropriate response time also motivated us to select these sensors. Table 1 presents the selected sensors.

Table 1. The selected sensors for our smart oven system.

\begin{tabular}{cccc}
\hline Sensor & Contextual Parameter & Technology & Response Time \\
\hline MICS 5521 & VOC & Metal Oxide Semiconductor & $10 \mathrm{~s}$ \\
TGS 2620 & Alcohol & Metal Oxide Semiconductor & $20 \mathrm{~s}$ \\
TGS 5042 & CO & Electrochemical & $60 \mathrm{~s}$ \\
MLX 90614 & Burner and Utensil Temperature & Infrared & $100 \mathrm{~ms}$ \\
HIH-5030 & Relative Humidity & Hygrometer & $5 \mathrm{~s}$ \\
SRF02 & Presence of Utensil on Burner & Ultrasonic & $72 \mathrm{~ms}$ \\
\hline
\end{tabular}

\section{Conclusions and Future Directions}

We presented in this paper the analytical study of sensors selection for our smart oven system. The study is based on analyzing the characteristics of the existing sensors to select the most appropriate. The selected sensor technologies include: infrared to measure burner and utensil temperatures, ultrasonic to detect the presence of a utensil on the oven burner, resistive hygrometer to measure the relative humidity, electrochemical to measure the concentration of $\mathrm{CO}$ gas, metal oxide semiconductor to measure the concentrations of VOC and Alcohol in the cooking smoke. We presented the results of testing each sensor in a real-world cooking environment and determined the capacities and the limitations of each sensor. The selected sensors have been used to build a prototype of a smart oven system [3,12].

As future directions, we aim at investigating more research to compensate the limitations and the measurement imprecision of each sensor and continuously improve the performance and the efficiency of the smart oven system by integrating the most recent advances in sensor technology.

Acknowledgments: We are grateful to all our students who have participated in this project, in particular Thomas Tessier. We are also grateful to Philippe Mabilleau.

Author Contributions: Rami Yared authored the paper, and Bessam Abdulrazak revised it and gave interesting remarks to improve its quality.

Conflicts of Interest: The authors declare no conflict of interest.

\section{References}

1. Abdulrazak, B.; Roy, P.; Gouin-Vallerand, C.; Belala, Y.; Giroux, S. Micro Context-Awareness for Autonomic Pervasive Computing. Int. J. Bus. Data Commun. Netw. (IJBDCN) 2011, 7, 49-69.

2. Yared, R.; Abdulrazak, B. Ambient Technology to Assist Elderly People in Indoor Risks. Computers 2016, 5, 22.

3. Yared, R.; Mallat, H.; Abdulrazak, B. Ambient Technology to Support Elderly People in Outdoor Risk Situations. In Information and Communication Technologies for Ageing Well and e-Health; Communications in Computer and Information Science; Springer International Publishing: Cham, Switzerland, 2015; Volume 578, pp. 35-56. 
4. Mallat, H.; Yared, R.; Abdulrazak, B. Assistive Technology for Risks Affecting Elderly People in Outdoor Environment. In Proceedings of the 1st International Conference (ICT4AgeingWell), Lisbon, Portugal, 20-22 May 2015.

5. Ahrens, M. Home smoke alarms: The data as context for decision. Fire Technol. 2008, 44, 313-327.

6. Lushaka, B.; Zalok, E. Development of a Sensing Device to Reduce the Risk from Kitchen Fires. Fire Technol. 2014, 50, 791-803.

7. Office of the Fire Marshal. Reducing Stovetop Fire; Fire Marshal's Public: Brockville, ON, Canada, 2009.

8. Alwan, M.; Dalal, S.; Mack, D.; Kell, S.; Turner, B.; Leachtenauer, J.; Felder, R. Impact of monitoring technology in assisted living: outcome pilot. IEEE Trans. Inf. Technol. Biomed. 2006, 10, 192-198.

9. Wai, A.A.P.; Devi, S.S.; Biswas, J.; Panda, S.K. Pervasive intelligence system to enable safety and assistance in kitchen for home-alone elderly. In International Conference on Smart Homes and Health Telematics; Lecture Notes in Computer Science LNCS; Springer: Berlin/Heidelberg, Germany, 2011; Voluem 6719, pp. 276-280.

10. Yuan, M.Y.; Green, J.R.; Goubran, R. Thermal imaging for assisted living at home: Improving kitchen safety. J. Med. Biol. Eng. 2013, 33, 380-387.

11. Yared, R.; Abdulrazak, B.; Tessier, T.; Mabilleau, P. Cooking risk analysis to enhance safety of elderly people in smart kitchen. In Proceedings of the 8th ACM International Conference on PErvasive Technologies Related to Assistive Environments (PETRA '15), Corfu, Greece, 1-3 July 2015.

12. Yared, R.; Abdulrazak, B. Toward context-aware smart oven to prevent cooking risks in kitchen of elderly people. In Information and Communication Technologies for Ageing Well and e-Health; Communications in Computer and Information Science; Springer International Publishing: Cham, Switzerland, 2015; Volume 578, pp. 57-77.

13. Abdulrazak, B.; Yared, R.; Tessier, T.; Mabilleau, P. Toward pervasive computing system to enhance safety of ageing people in smart kitchen. In Proceedings of the 1st International Conference (ICT4AgeingWell), Lisbon, Portugal, 20-22 May 2015; pp. 17-28.

(c) 2016 by the authors. Licensee MDPI, Basel, Switzerland. This article is an open access article distributed under the terms and conditions of the Creative Commons Attribution (CC BY) license (http://creativecommons.org/licenses/by/4.0/). 